\title{
Sport Training Using Body Sensor Networks: A Statistical Approach to Measure Wrist Rotation for Golf Swing
}

\author{
Hassan Ghasemzadeh, Vitali Loseu, Eric Guenterberg, Roozbeh Jafari \\ Embedded Systems and Signal Processing Lab, Department of Electrical Engineering \\ University of Texas at Dallas, Richardson, TX 75080, United States \\ \{h.ghasemzadeh, vitali.loseu, mavpion, rjafari\}@utdallas.edu
}

\begin{abstract}
Athletes in any sports can greatly benefit from feedback systems for improving the quality of their training. In this paper, we present a golf swing training system which incorporates wearable motion sensors to obtain inertial information and provide feedback on the quality of movements. The sensors are placed on a golf club and athlete's body at positions which capture the unique movements of a golf swing. We introduce a quantitative model which takes into consideration signal processing techniques on the collected data and quantifies the correctness of the performed actions. We evaluate the effectiveness of our framework on data obtained from four subjects and discuss ongoing research.
\end{abstract}

\section{Keywords}

Sport Training, Body Sensor Networks, Quantitative Analysis, Golf Swing

\section{INTRODUCTION}

Sport training represents the body's adaptation to conditions of certain exercises. One can achieve considerable progress in a sport with the aid of appropriate exercises and training methods [1]. The quality and organization of the training typically influences degree of final proficiency and the speed with which this proficiency is achieved. Feedback becomes essential for training when new plans are structured in order to make incremental progress by practicing sport skills.

Sport-specific coaching systems have allocated increasing interest lately, leading to the development of frameworks which are capable of acquiring and processing physiological and behavioral variables for a given sport. Advancements in microelectronics and wireless communication have enabled the design of light-weight embedded sensory devices [2]. Ability of wireless sensor platforms to perform computations, store necessary data, and communicate within a short range make them attractive for the development of wearable

Permission to make digital or hard copies of all or part of this work for personal or classroom use is granted without fee provided that copies are not made or distributed for profit or commercial advantage and that copies bear this notice and the full citation on the first page. To copy otherwise, to republish, to post on servers or to redistribute to lists, requires prior specific permission and/or a fee.

BodyNets '09 April 1-3, 2009, Los Angeles, CA, USA.

Copyright 2008 ICST 978-963-9799-41-7. systems. In particular, sport training systems aim to incorporate either off-body devices placed in the environment or a composition of embedded devices within the sports equipment and on-body sensors. In this paper, we investigate how such a coaching system can be designed to provide feedback to novice golf players.

The popular sport of golf requires a complicated sequence of motions to swing the golf club properly with the primary goal of propelling the golf ball a certain distance in a desired direction. A proper golf swing can make the difference between a long straight ball flight and a shorter hook or slice as a result of an improper swing. A repeatable and consistent golf swing can also dramatically improve a golfer's score. However, this single movement which has such a major impact on the player's overall game is difficult to master and execute consistently for players who are new to the sport or have little experience.

To make matters worse, certain variables, such as which club is used, where the player wants to place the ball on the golf course, and the current wind conditions cause how the swing is executed from one swing to the next. To resolve all of these complications, players who are serious about their game seek out instructors, golf instructional books, or other training aids to help them to obtain the "perfect" golf swing. These players can potentially benefit from wearable coaching systems where information on the quality of the performed swing can be provided in real-time. Such a system must be mobile to be usable at the location where the sport takes place.

In this paper, we describe a system that qualitatively analyzes the golf driver swing using a body sensor network (BSN). We describe the design requirements and the information processing flow for a golf swing training system capable of detecting mistakes new players make in executing a good golf swing. Our system is developed to assess the quality of a golf swing with respect to incorrect movements. Wrist rotation is among most common mistakes in golf, which causes the ball to fly either to the right or left of the target line. We place embedded sensor nodes on the player's upper body and the golf club to monitor the acceleration and angular velocities of those points during the swing. The system can be used to assist the player in developing a correct swing in four major segments of golf swing: takeaway, backswing, downswing and follow-through. The sensor nodes collect data for the sequence of actions in a swing which is then preprocessed locally to facilitate subsequent in-network operations. The data is then sent to a base station for further analysis. At the base station, the qual- 


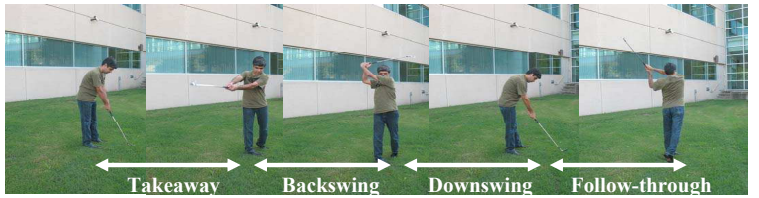

Figure 1: Golf swing as a four-phase motion

ity of each segment is expressed as the amount of deviations from target line. Our experiments demonstrate that our system is capable of quantifying a golf swing with respect to wrist rotation. This information can then be provided as feedback to the player to help them pinpoint which parts of their swing may need practice. This information can also help the player determine if any improvement was achieved.

\section{PRELIMINARIES}

In order to effectively analyze performance of a golfer, a precise understanding of the complicated movements involved in the golf swing is required. We use a typical model of the golf swing which splits a complete swing into several major parts [3], [4], [5]. The model helps in determining significant factors of improper actions. In the following, we present our model of the swing along with common mistakes that lead to a bad swing.

\subsection{Golf Swing Model}

A full swing is a complex motion of the body aimed at accelerating the club at great speed. The motion starts at an initial position, referred to as the address position [6], followed by the swing. A golf swing can be divided into smaller segments. Our sport training system is based on a golf swing model which considers a full swing composed of four major segments: takeaway, backswing, downswing, and follow-through [5]. Takeaway starts as the first movement after the address position and ends when the club is approximately parallel to the target line and at waist level. The backswing follows the takeaway and continues until the golf club is lifted to its highest point behind the player. Following this is the downswing in which the club is brought back down to hit the ball. After impact with the ball, the follow-through motion brings the club to its stopping point in front of the player. These segments are demonstrated in Figure 1.

The choice of the aforementioned model simplifies evaluation of the movement by breaking a complex motion into less complicated actions. It further enables more precise analysis of the actions by reporting the quality of each individual part of the swing. Furthermore, the data obtained by our system verifies that each segment can be specified by particular patterns in the signal. We will provide the evidence later in this paper.

\subsection{Fundamental Guidelines}

For a golf player to develop a sound swing, it is required to know principles that are essential to building a prefect swing. Applying these fundamental guidelines helps individual golfers improve their proficiency by learning how to establish positions as well as how to adjust those parts of the swing that is not fundamentally correct. The goal in achieving a perfect swing is to hit the ball squarely and straight [7]. This would also give the golfer maximum distance. Con- sequently, it is important to investigate actions that prevent development of a perfect swing. According to the literature, there are two kinds of common mistakes new players make resulting in a poor shot [6]: wrist rotation and out-ofplane movements. In this study, we focus on evaluating golf swing in terms of the angle of wrist rotation. However, we make our experiments highly controlled to prevent introducing effects of other mistakes, e.g. out-of-plane movements, in our results. Wrist rotation occurs when the player rotates the wrists clockwise or counterclockwise resulting in the golf club to become "open" or "closed," respectively [6]. This can happen during any segment of the swing. The result is that at impact, the golf ball will go either to the right or the left of the target line. Hitting the ball with an open clubface will cause the ball to fly to the right of the target line (slice), while hitting the ball with a closed clubface will cause the ball to fly to the left of the target line (hook). Both of these outcomes are highly undesirable when playing a game and result from the rotation of the wrist.

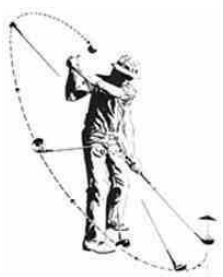

\section{Figure 2: Swing plane}

The second common swing mistake is out-of-plane movement. The golf swing plane is defined by the plane which contains the line created by the golf club at address and the target line. This is demonstrated in Figure 2 which is adopted from [8]. A swing is considered an in-plane swing if the swing, including takeaway, backswing, downswing, and follow-through, remains on a plane at the address position [6]. Out-of-plane movements can happen during any segment of the swing. They can be due to several important movements and postures including over-bending the elbows, raising the arms too high, not raising the arms enough, and bending the wrists among others. Each of these actions has a different effect on the outcome of the swing. In general, outof-plane movements cause the ball to leave the target line, but more importantly, they reduce strength of the swing resulting in a weak impact and shorter driving distance.

\section{SYSTEM ARCHITECTURE}

We use a BSN consisting of several sensor units placed on the body and the golf club to capture the physical movements of the golf swing. Each sensor node, also called a mote, is equipped with a custom-designed sensor board consisting of several inertial sensors as shown in Figure 3. We use the TelosB mote which is commercially available from XBow $\AA$. The mote has a microcontroller for processing and storage, and a radio for communication. Embedded with our custom-designed sensor board, a tri-axial accelerometer and a bi-axial gyroscope are interfaced with the mote platform. The mote and the sensor board are powered by a Li-Ion battery integrated with each node.

Our body-worn sensor nodes are placed on the upper body and arms to capture significant motions during the swing 


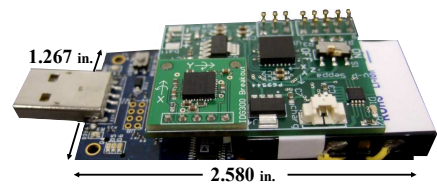

Figure 3: Custom-designed sensor board attached to a mote

[9]. The movements of the golf club are captured by the two nodes attached to the club. This configuration ensures that the system captures inertial information associated with the major parts of the body involved in the golf swing. We placed two nodes on the golf club (one near the club head and another near the grip, as shown in Figure 4), one on the right wrist, one on the left arm, and one on the back at waist level. We will demonstrate effectiveness of this sensor setup through our experiments. The optimal sensor configuration, including the best senor placement and smallest set of sensors required for our system, is not investigated in this paper.

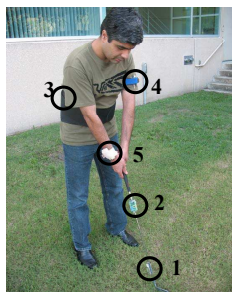

Figure 4: Experimental subject and processing nodes with motion sensors

The processing unit of each node samples sensor readings at $50 \mathrm{~Hz}$. This tentative sampling rate is experimentally chosen to provide sufficient resolution while compensating for bandwidth constraints of our sensor platform. We will investigate more efficient rates later in this paper. Each sensor node can perform local processing on the inertial data and transmit the result wirelessly to a base station. The base station can be either another mote or a PDA which collects local information from all other nodes, performs final processing, and provides the user with a feedback on the quality of the swing.

\section{SIGNAL PROCESSING}

Our model for assessing quality of golf swings aims to utilize processing capability of each sensor node and combine local information obtained from all sensor nodes to achieve a measure of quality. This process consists of several steps as illustrated in Figure 5 and explained below.

The preprocessing consists of filtering and segmentation to facilitate subsequent operations without losing relevant information. The data collected at each sensor node is locally filtered. We use a five-point moving average filter to reduce the effect of noise. The number of points used to average the signal is experimentally chosen to maintain sharp step response while a smooth output signal can be obtained. For segmentation, we determine parts of the signal that represent swing segments. That is, each signal segment corresponds to one of takeaway, backswing, downswing and

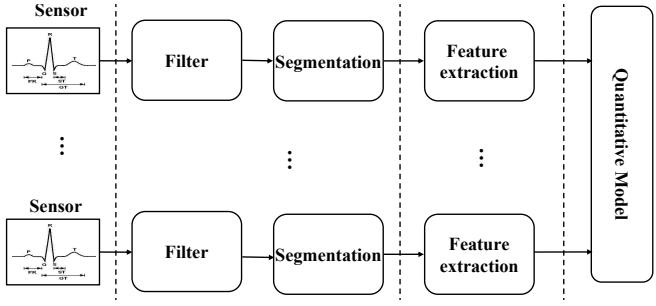

Figure 5: Signal processing blocks for quality analysis of golf swings

follow-through. Currently, we perform this process manually to avoid introducing errors by automatic segmentation. We take advantage of video which captures experimental procedure to perform fine-grain manual segmentation. This video is specifically used in our prototype to isolate and identify segments of the golf swing.

In feature extraction, an exhaustive set of features is considered to ensure capturing as much useful information as possible for each movement segment. We extract an exhaustive set of time-domain features including statistical and morphological features. Each statistical feature is a mathematical function taken over a complete segment. Morphological features, however, are calculated from $m$ uniformly distributed samples over a complete segment.

The quantitative model performs further analysis on the features extracted from all sensor nodes in order to obtain a quality metric. The quality of each segment of a golf swing can be measured with respect to different criteria. Examples of such criteria include the amount of wrist rotation and how out of plane a swing is. We develop our model for quantifying golf swings with respect to several criteria. Our model employs feature conditioning techniques to refine features contributing to the quality of the swing. Although the exhaustive set of features maintains relevant information on the quality the swing, it contains relatively large number of redundant features. On the other hand, curse of dimensionality [10] is an impediment for our system as our sensor nodes are constrained in terms of computational capabilities, communication bandwidth and memory. A high dimensional feature space requires more bandwidth for transmission and more computation for quality analysis. Furthermore, quality of a golf swing with respect to each criterion can be expressed by certain properties of the physical movement. Therefore, specific tools are required to extract such attributes from the signal. We use several signal processing techniques including PCA (Principal Component Analysis) [11] and LDA (Local Discriminant Analysis) [12] to obtain significant information with respect to each criterion.

\section{QUANTITATIVE MODEL}

Our technique of quality analysis takes advantage of linear projection methods traditionally used in the field of signal processing and pattern recognition [12]. The intuition behind this model is that the quality of the golf swing with respect to a specific criterion is proportional to the quality of physical movement. That is, the deviation of the swing should be linearly related to our quality metric. When assessing the system with respect to special criterion, e.g. wrist rotation, the degree of improperness can be exclusively quantified by a subset of features. Our technique aims to find features that are unique for each particular target qual- 
ity metric and maintain linearity of that metric. Inspired by linear methods of LDA and PCA, we build a quality measure for every given criterion by further processing of features as illustrated in Figure 6.

The set of features extracted from observations across the network are fed to the data fusion block to form a higher dimensional feature space. Let $F_{1}, F_{2}, \ldots, F_{n}$ be feature vectors of size $N \times m$ obtained from sensor nodes $\{1,2, \ldots, n\}$ where $N$ denotes the number of observations and $m$ represents the number of features. The new feature vector $F$ has a size of $N \times M$ where $M=n \times m$.

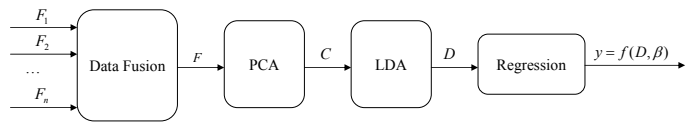

Figure 6: Building quantitative model

PCA, known as an effective dimension reduction techniques [13], aims to replace the original features with a new set of variables that can be ranked in the order of their importance. The first few principal components account for those projections of the feature space that provide most of the information in the data. This technique is widely used for dimension reduction where a high-dimension dataset is replaced with a new dataset with fewer features. The resulting projections are given by $C=\left[C_{1}, \ldots, C_{L}\right]$ where each new feature $C_{i}$, called a principal component, can be expressed by a linear combination of original features $\left[f_{1}, \ldots, f_{M}\right]$ :

$$
C_{i}=a_{i 1} f_{1}+a_{i 2} f_{2}+\ldots+a_{i M} f_{M} \quad \forall \mathrm{i} \in\{1, \ldots, \mathrm{L}\}
$$

where $a_{i j}$ are determined by eigenvalue decomposition on the original feature space.

LDA, used for both classification and dimension reduction, is characterized as trace optimization on scatter matrices [14]. The technique aims to maximize the between-class scatter while minimizing within-class scatter. It selects the feature vectors given by

$$
D=\underset{D}{\arg \max } \operatorname{trace}\left(\frac{D^{T} S_{b} D}{D^{T} S_{w} D}\right)
$$

where $S_{b}$ denotes the between-class scatter matrix and $S_{w}$ represents within-class scatter matrix. Classical LDA suffers from Small Sample Size (SSS) [15] problem, that results in singularity of the within-class scatter matrix. One way to overcome the singularity of $S_{w}$ is to use PCA to reduce the dimension of the original dataset before applying LDA. The technique is known as subspace LDA [16]. We use this method to refine the feature space prior to using LDA. We set the number of principal components, $L$, fed to the LDA block to be equal to the rank of the between-class scatter matrix $S_{w}$.

Let $X$ be a given dataset of size $N \times M$ where $N$ is the number of observations and $M$ is the number of features. For every criterion for which our system tends to build a qualitative model, we assume that the dataset is divided into $k$ groups $g_{1}, g_{2}, \ldots, g_{k}$ each accounts for a particular degree of quality with respect to the given type of bad swing. The reduced feature space $C$ which has a size of $N \times L$ is applied to the LDA module to obtain $k-1$ projections. The projections $D=\left[D_{1}, \ldots, D_{k-1}\right]$ from LDA, also called

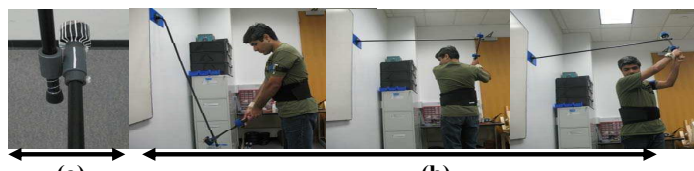

(a)

(b)

Figure 7: Experimental setup for wrist-rotation

discriminant functions, give directions that maximize the distance between different groups and minimize distances between trials within each group.

$$
\begin{aligned}
D_{i}=b_{i 1} C_{1}+b_{i 2} C_{2}+\ldots+b_{i k-1} C_{k-1} \\
\forall i \in\{1, \ldots, k-1\}
\end{aligned}
$$

Although the first projection obtained from LDA provides maximum discrimination, the groups may partially overlap if only this projection is considered as our evaluation metric. To take maximum discrimination into consideration, we build a regression model according to the LDA projections. This model is given by (4).

$$
y_{i}=\beta_{0}+\sum_{j=1}^{k=1}\left(\beta_{j} D_{i j}+\alpha_{j} D_{i j}^{2}\right)+\varepsilon_{i} \quad i=\{1, \ldots, N\}
$$

where the dependent variable $y_{j}$ is a linear combination of parameters $\beta_{i j}$ and $\alpha_{i j}$, and dependent variables $D_{i j}$ refer to the discriminant function $D_{j}$ associated with $i-t h$ observation.

The qualitative model can be tested by computing various statistics that measure the difference between the predicted values, $\widehat{y}_{i}$, and the expected values, $y_{i}$. The Root Mean Squared Error (RMSE) and Mean Absolute Error (MAE) are among most common statistics used to evaluate the overall quality of a regression model. RMSE is the square root of the average squared distance of data point from the fitted line and is given by

$$
R M S E=\sqrt{\frac{1}{N} \sum_{j=1}^{N}\left(y_{i}-\widehat{y}_{i}\right)^{2}}
$$

where $\mathrm{N}$ denotes cardinality of the validation set. MAE is the average of the absolute value of the residuals and is given by

$$
M A E=\frac{1}{N} \sum_{j=1}^{N}\left|y_{i}-\widehat{y}_{i}\right|
$$

\section{QUANTITATIVE ANALYSIS FOR WRIST ROTATION}

In this section, we investigate quality measure with respect to the wrist rotation. We find a linear projection of feature space that monotonically changes while the angle of rotation varies. This projection is obtained by LDA and is fed to a regression model to quantify the degree of improperness of movement. The system requires a dataset with trials obtained from several variations of the wrist rotation. Swings performed for each variation are required to account for consistent physical movements. That is, within each group $g_{i}$, the trials should have the same degree of wrist rotation while different groups exclusively differ with respect to the rotation. This would imply the need for a highly controlled experimental environment. Therefore, we use a home swing trainer [17] [18] which helps to maintain an in-plane swing. The device has a rigid rod with one end mounted 
on the wall and the other end on which the golf club slides as shown in Figure 7. The rod and the club are connected at one end as illustrated in Figure 7.a where the club can rotate along its axis. We modified the club to ensure that it was fixed about its axis during every trial. This makes our experiments even more controlled because the amount of wrist rotation remains consistent provided that the subject maintains a steady grip throughout each trial. The club is marked at both head and grip sides to depict the angle of rotation. Hence, by placing sensor nodes at locations associated with angles, a consistent angular wrist rotation is maintained.

An extremely important requirement in building our model is to restrict each trial to a specific type of bad swing. The experimental setup for the wrist rotation, as shown in Figure 7.b, keeps all segments of a swing within the same plane resulting in maintaining an in-plane swing. This ensures that different trials can differ only in the amount of the wrist rotation. The resulting model then will be able to quantify incorrect swings in terms of angular rotation. In reality, however, several types of mistakes can be made independently by the golfer, each of which must be quantified using individual models. Integrating evaluation of mistakes other than wrist rotation into our existing model is a problem that we will investigate in the future.

To build the quantitative model for wrist rotation, $N$ number of observations associated with $k$ different variations of wrist rotation are required. The data collected for $k$ angles form a dataset of groups $g_{1}, g_{2}, \ldots, g_{k}$. The LDA projection can be derived as described previously. Finally, the parameters of a linear regression are calculated based on $N$ values of the discriminant functions introduced by LDA

\section{EXPERIMENTAL VERIFICATION}

In this section, we describe our methods of data collection, model generation and validation to provide feedback on the quality of movements with respect to wrist rotation.

\subsection{Experimental Procedure}

We conducted our experiments to express the quality of the golf swing with respect to the wrist rotation. The experiments were conducted on three male subjects and one female subject all aged between 20 and 35. Each of the subjects wore three on-body sensors. In addition, two sensor nodes were placed on the golf club: one on the club head and one on the grip as shown in Figure 4. The subjects were asked to perform the golf swing ten times for each of the variations listed in Table 1 .

Table 1: Experimental movements

\begin{tabular}{ccl}
\hline No. & Angle & Description \\
\hline 1 & 0 & Perfect Swing \\
2 & +20 & Twenty degrees clockwise \\
3 & +40 & Forty degrees clockwise \\
4 & +60 & Sixty degrees clockwise \\
5 & +80 & Eighty degrees clockwise \\
6 & -20 & Twenty degrees counter clockwise \\
7 & -40 & Forty degrees counter clockwise \\
8 & -60 & Sixty degrees counter clockwise \\
9 & -80 & Eighty degrees counter clockwise \\
\hline
\end{tabular}

Our subjects performed swings after first addressing the ball with $20^{\circ}, 40^{\circ}, 60^{\circ}$ and $80^{\circ}$ clockwise and counter-clockwise rotation of the wrists. Each subject also performed a perfect golf swing that has no wrist rotation or out-of-plane movements. For each movement, the amount of wrist rotation was controlled by fixing the location of the nodes placed on the golf club. The subjects must grip the club aligned with the nodes on the club. They were asked to keep their wrist fixed throughout the movements. This allows the system to control the swing plane while achieving consistent angles in different segments of the swing. All of the swings were performed in the absence of a golf ball. The subjects were also asked to perform the swings at a specified speed for experimental consistency.

Table 2: Statistical and morphological features

\begin{tabular}{ccl}
\hline No. & Symbol & Description \\
\hline 1 & Amp & $\begin{array}{l}\text { Different between maximum amplitude } \\
\text { and mean of signal segment }\end{array}$ \\
2 & Med & Median value of signal segment \\
3 & Min & Minimum value of signal segment \\
4 & Max & Maximum amplitude of signal segment \\
5 & Mean & Mean value of signal segment \\
6 & P2P & Peak-to-Peak amplitude of signal segment \\
7 & Var & Variance of signal segment \\
8 & Std & Standard deviation of signal segment \\
9 & RMS & Root Mean Square of signal segment \\
10 & $\mathrm{~S} 2 \mathrm{E}$ & Start-to-End value of signal segment \\
11 & Slope & First derivative of signal segment \\
12 & $\mathrm{~V}$ & Value of morphological point \\
13 & $\mathrm{~T}$ & Time of morphological point \\
14 & $\mathrm{dx}$ & First derivative \\
15 & $\mathrm{~d} 2 \mathrm{x}$ & Second derivative \\
\hline
\end{tabular}

An extra mote was connected to a laptop via USB port to collect data from all sensor nodes. The data was collected using our tool developed in MATLAB. We followed the procedure for data collection, preprocessing, feature extraction, model generation, and validation as described previously. We processed collected data offline using our tools developed in MATLAB

\subsection{Quantification Results}

For each trial, the data collected from four subjects was first preprocessed using a five-point moving average filter to remove the effect of noise. Each trial was divided into four major segments consisting of takeaway, backswing, downswing and follow-through. The manual segmentation was performed with the help of the video recorded during data collection. An exhaustive set of features was extracted from each segment. The features include statistical and morphological features as shown in Table 2 in which the first eleven features represent statistical features obtained from each signal segment, and the next four features are morphological features extracted from ten evenly distributed samples over each segment. We used $50 \%$ of the trials for the training to build our quantitative model, and the rest to evaluate performance of the model.

For each of the major segments, a separate quantitative model was built. The features extracted from five sensors (x,y,z accelerometer, and $\mathrm{x}, \mathrm{y}$ gyroscope) formed a $215 \mathrm{di}-$ mensional feature space for each sensor node. Data fusion was used to combine features from all sensor nodes to form a 1075 dimensional feature space which was used for subsequent processing. The features were fed to the PCA block for dimension reduction. Only a small number of principal components obtained from PCA were used to find LDA projections. The number of principal components was set to the rank of the within-class scatter matrix.

Given nine different groups of wrist rotation, LDA creates eight discriminant functions in the form of linear combinations of the input. In Figure 8 we illustrate projections of the training trials using the first two dimensions for takeaway, 


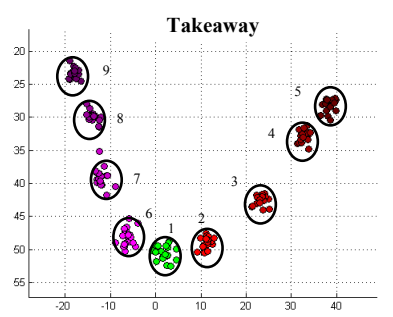

Downswing
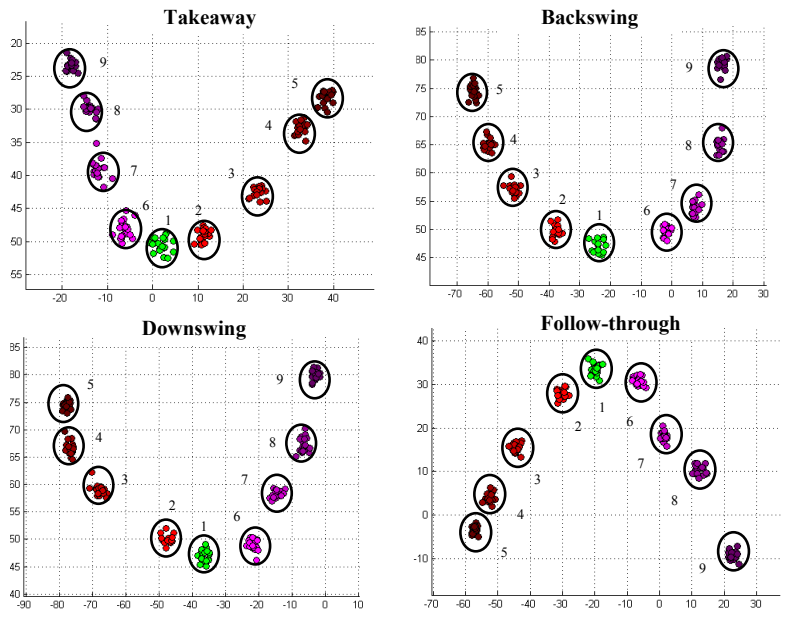

Figure 8: Projection of training trials for different swing segments using LDA

backswing, downswing and follow-through. The group 1 indicated by green color corresponds to perfect swings while red represented by groups $2,3 \ldots 5$ and magenta colors annotated by $6,7 \ldots 9$ show clockwise and counter clockwise rotations respectively. These figures demonstrate the effectiveness of our technique in distinguishing different variations of the wrist rotation. Furthermore, the graphs would clearly describe the angular rotation.

The projections obtained by applying LDA were used to build a linear regression as described previously. We used the validation set to measure the degree of wrist rotation based on the model acquired. The values of error in terms of RMSE and MAE are shown in Table 3 and Table 4 respectively. In overall, the amount of root mean squared error was 15.5, 10.7, 8.9 and 9.1 for takeaway (TA), backswing (BS), downswing (DS) and follow-through (FT) respectively. The overall value of absolute mean error was reported as 9.2 , 7.7, 6.6 and 6.5 degrees for TA, BS, DS and FT respectively which introduces an average error of less than 10 degrees for all segments.

Table 3: RMSE values for different swing segments

\begin{tabular}{ccccc}
\hline Group & TA & BS & DS & FT \\
\hline$g_{1}$ & 8.3 & 6.1 & 5.0 & 7.4 \\
$g_{2}$ & 13.8 & 14.8 & 15.4 & 7.3 \\
$g_{3}$ & 30.9 & 8.1 & 5.8 & 6.4 \\
$g_{4}$ & 12.8 & 10.4 & 4.9 & 11.6 \\
$g_{5}$ & 19.5 & 15.0 & 9.8 & 11.6 \\
$g_{6}$ & 8.5 & 7.3 & 7.1 & 5.6 \\
$g_{7}$ & 13.5 & 8.6 & 7.9 & 11.4 \\
$g_{8}$ & 12.0 & 7.6 & 9.9 & 7.0 \\
$g_{9}$ & 5.6 & 13.6 & 9.7 & 10.9 \\
\hline Overall & 15.5 & 10.7 & 8.9 & 9.1 \\
\hline
\end{tabular}

Table 4: MAE values for different swing segments

\begin{tabular}{ccccc}
\hline Group & TA & BS & DS & FT \\
\hline$g_{1}$ & 5.7 & 5.0 & 4.5 & 7.0 \\
$g_{2}$ & 12.3 & 10.7 & 10.8 & 5.4 \\
$g_{3}$ & 14.3 & 5.8 & 4.6 & 5.0 \\
$g_{4}$ & 10.9 & 8.0 & 4.3 & 9.3 \\
$g_{5}$ & 16.3 & 12.1 & 8.0 & 8.3 \\
$g_{6}$ & 6.9 & 5.2 & 5.7 & 3.8 \\
$g_{7}$ & 9.9 & 7.0 & 6.9 & 6.8 \\
$g_{8}$ & 9.5 & 6.0 & 7.4 & 5.6 \\
$g_{9}$ & 4.5 & 9.6 & 7.3 & 8.3 \\
\hline Overall & 9.2 & 7.7 & 6.6 & 6.5 \\
\hline
\end{tabular}

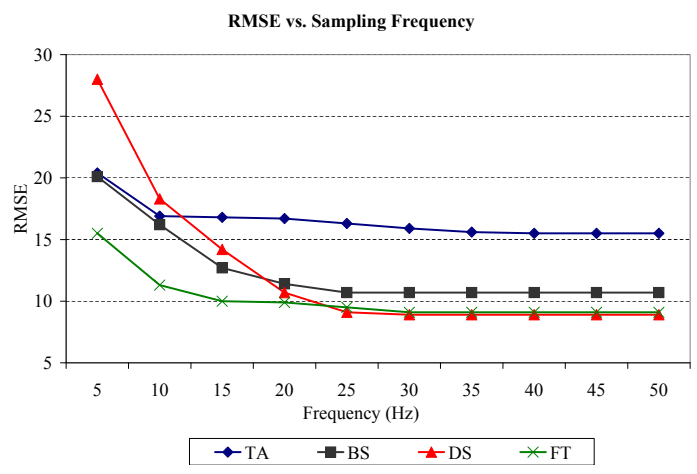

Figure 9: Root mean squared error vs. sampling frequency for different segments

\subsection{Frequency Adjustment}

Throughout our experiments, we used a sampling frequency of $50 \mathrm{~Hz}$ which provides good resolution in capturing motions of golf swing. Reducing the sampling frequency can potentially reduce the complexity of processing. However, over-reduction may eliminate important details of the signal. In an effort to address this issue, we further adjusted our sampling rate with respect to the performance of our model. Recall the performance of our model expressed in terms of RMSE and MAE, our adjustment process tends to find a minimum sampling frequency that maintains approximately similar performance to $50 \mathrm{~Hz}$.

For the purpose of frequency adjustment, we measured RMSE and MAE errors for different sampling frequencies between $5 \mathrm{~Hz}$ and $50 \mathrm{~Hz}$. The results are illustrated in Figure 9 and Figure 10. For each segment, the error remained almost constant beyond certain frequency. This threshold varied from one segment to another. The lowest threshold was obtained for takeaway $(10 \mathrm{~Hz})$ and the highest frequency belonged to downswing and follow-through $(30 \mathrm{~Hz})$. The difference between minimum sampling frequencies is mainly a factor of changes in speed of swing motions from one segment to another. According to the analysis performed using high speed cine-films of tournament professionals [6], the golf club can move four times faster during downswing than it usually does during takeaway and backswing. As a result, faster motions require higher sampling frequencies to ensure the collected data has acceptable resolution. Considering the worst case (i.e. frequency required for downswing and follow-through), our system allows a frequency of $30 \mathrm{~Hz}$ while maintaining the same amount of error as reported at $50 \mathrm{~Hz}$.

\section{RELATED WORK}

Use of inertial sensors in BSNs is motivated by biomedical applications such as fall detection, gait analysis, sport medicine and balance assessment, and has received much attention during recent years. Authors in [19], [20], [21], and [22] introduce a framework for human action recognition using motion sensors. They integrate on-body sensors including accelerometers and gyroscopes in a wireless sensor network to classify physical movements. Maithe et al. [23] use a tri-axial accelerometer mounted on the waist to recognize basic daily movements using a hierarchical classification scheme. A pattern recognition technique for evaluating the 


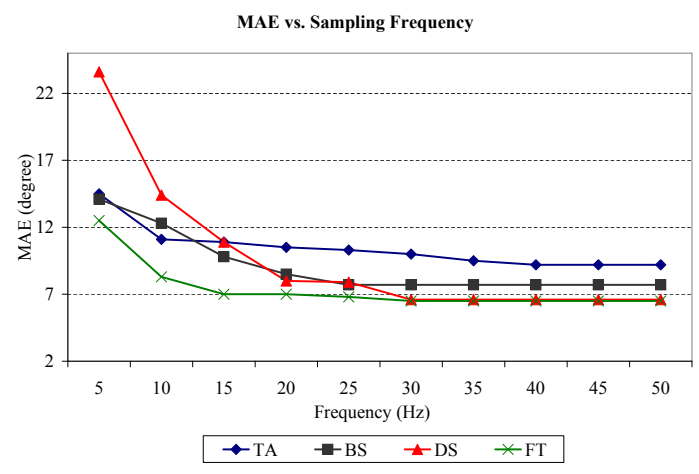

Figure 10: Mean absolute error vs. sampling frequency for different swing segments

performance of the human postural control system using inertial and EMG sensors is presented in [24].

Advances in technology has enabled design of sports feedback systems which accelerate training by providing students with information regarding mistakes made during practice. In [25], Spelmezan et al. present an on-body wireless sensor platform for real-time snowboard training. They deploy inertial sensor, bend sensors and force-sensitive resistors along with communication facilities in a wireless network to capture and analyze rider's motion and posture on the snowboard. The trajectory of the swing is then used to provide qualitative results. Golnalez et al. [26] and Alvarez et al. [27] study the problem of step estimation which is an important issue in designing coaching systems. Despite their successful development of sports training systems, unique complexities in the golf movements make the aforementioned techniques inappropriate for a golf swing trainer.

A variety of golf swing analysis aids have become popular recently, making use of technologies such as high-speed photography, inertial sensors, and motion capture using magnetic, radio frequency, or ultrasonic markers. These systems can incorporate either devices placed in the sports environment [28] [29] or sensors embedded within the sports equipment and human body [4] [30]. In [28], Urtasun et al define a temporal motion model that allows them to accurately extract $3 \mathrm{D}$ golf swing motion from a single camera while no markers are required to be placed on the subject. The model allows them to overcome the obstacles of subject self-occlusion and movements which are perpendicular to the camera plane. This approach simplifies the computational complexity typical in motion capture by using only one camera instead of many. In [31], Betzler et al describe the application and limitations of 3D motion analysis in measuring golf swing motion. Golf-specific limitations of 3D motion analysis include the high velocity of the hands, club and ball; inaccuracies in determination of body segment rotations; vibration of markers at impact; and marker placement and occlusion. The authors designed a test setup to minimize the effect of these errors by using cameras and careful placement of several markers on the golfer and golf club. Kiat et al. [32] present a different approach to measure golf-swing motion. They place electrogoniometers along the left arm and utilize dual Euler angles and dual Euler velocity analysis to estimate the location and velocity of the club head. This method is able to provide details on the indi- vidual segmental contributions of the left arm to the final swing movement. The calculated club head path is verified by comparing those obtained through video analysis. In [33], inertial measurement units (IMU) were placed at the grip end of a golf club to measure acceleration and angular velocities with six degrees-of-freedom for a golf putting training system. A putting robot capable of performing highly repeatable putts and independent measuring instruments as used to assess the accuracy of the sensor system. By using measurement theory, their system was able to provide the position of the club head to within $3 \mathrm{~mm}$ and the orientation of the clubface to within $0.5^{\circ}$. Golf training aids have also been proposed in [34], [35], and [36] which target specific problems faced by novice golfers. A swing guide is presented in [34] which aims to help the golfer coordinate the movements performed during a swing. The exercises the author recommends while using the device target the coordinated movement of the hips, shoulders, elbows, wrists, and the golf club. A mechanical golf swing training device is presented in [35] to help players perfect their backswing and downswing movements. The training device helps players focus on the non-dominant arm and shoulder while keeping the swing in the proper swing plane. The proposed device helps players to develop muscle memory in their upper body to produce a smooth, consistent, and controllable swing. Another training device in [36] helps players to maintain proper right leg positioning during the backswing. Its purpose is to restrict the lateral movement of the right leg away from the target during the backswing while not hindering the forward movement of the legs during downswing and follow-through. In [8], the authors model the golf swing as a double pendulum system. Wireless inertial sensors are placed along the body and golf club to determine how closely the movements of the body follow predetermined motion rules. This is used as a quality measure for the golf swing. The authors define a physical model of the swing which accounts for the length of the backswing, the wrist-cock angle, energy transfer during the swing, the swing plane, and club-head speed.

Though most of the above training systems are successful in introducing methods for golf swing analysis, the training aids can only be accessed at a specialized facility making widespread deployment difficult. Properties such as mobility and wearability make BSNs more promising for designing sport feedback systems. We take advantage of pattern recognition techniques in designing our training system to avoid the need for per-joint and complementary sensor deployment as required for kinematic analysis techniques.

\section{DISCUSSION AND FUTURE WORK}

Our quantitative model functions based on feature vectors from all sensor nodes across the network. With an exhaustive set of features obtained from each segment, this may yield in large volume of data for signal processing and communication. The amount of data required for signal processing, however, can be significantly reduced by the data reduction techniques described earlier. Since each sensor node partially contributes to the linear projections of PCA and LDA, it can combine local features using pre-obtained eigenvectors and transmit a single value for each trial to the base station.

At this stage of our study, we process data offline. This is convenient for rapid prototyping and algorithm development. However, we have great suspicion that our algorithms 
for signal processing can be implemented and executed on the motes.

In this research, we focused on building our quantitative model for non-ideal movements due to wrist rotation. Evaluating this model for other types of incorrect swings requires controlling experiments for those types of errors. We plan to investigate this in future.

\section{CONCLUSIONS}

In this paper, we presented a system which uses body sensor networks for the purpose of golf swing training. We developed a quantitative model which can provide feedback on quality of movements for the purpose of training. The system architecture, signal processing methods and experimental results of the system were presented. The results demonstrate that our model is able to provide information on the quality of a golf swing with respect to the angle of the wrist rotation.

\section{Acknowledgments}

The authors would like to thank Dr. Michael Motes of Center for BrainHealth, UT-Dallas for his valuable input concerning development of the experimental protocol. The authors would also like to thank Ville-Pekka Seppa for his efforts in designing the sensor board.

\section{REFERENCES}

[1] A. Viru and M. Viru, Biochemical Monitoring of Sport Training. Human Kinetics, 2001.

[2] L. Benini, E. Farella, and C. Guiducci, "Wireless sensor networks: Enabling technology for ambient intelligence," Microelectronics Journal, vol. 37, no. 12, pp. 1639-1649, 2006

3] D. Kim, P. Millett, J. Warner, and F. Jobe "Shoulder Injuries in Golf," American Journal of Sports Medicine, vol. 32, no. 5 , p. 1324,2004

[4] K. Watanabe and M. Hokari, "Kinematical analysis and measurement of sports form," Systems, Man and Cybernetics, Part A, IEEE Transactions on, vol. 36, no. 3, pp. 549-557, May 2006.

[5] P. A. Hume, J. Keogh, and D. Reid, "The role of biomechanics in maximising distance and accuracy of golf shots," Sports Medicine, vol. 35, pp. 429-449(21), 2005.

[6] A. Cochran, J. Stobbs, and G. S. of Great Britain, Search for the Perfect Swing. Triumph Books, 1968.

[7] J. Tartagni and P. Schmid, "Putting stroke training aid," Jan. 6 2004, uS Patent 6,672,974.

[8] D. K. Arvind and A. Bates, "The speckled golfer," in BodyNets '08: Proceedings of the ICST 3rd international conference on Body area networks. ICST, Brussels, Belgium, Belgium: ICST (Institute for Computer Sciences, Social-Informatics and Telecommunications Engineering), 2008, pp. 1-7.

[9] M. Bellagamba, "Athletic swing practice apparatus," Jun. 18 1991, uS Patent 5,024,443.

[10] D. Donoho, "High-dimensional data analysis: The curses and blessings of dimensionality," Society Conference on Math Challenges of the 21st Century, 2000.

[11] I. Jolliffe, "Principal component analysis," 2002.

[12] R. Duda, P. Hart, and D. Stork, Pattern classification and scene analysis. Wiley New York, 1973.

[13] J. Yu, Q. Tian, T. Rui, and T. Huang, "Integrating Discriminant and Descriptive Information for Dimension Reduction and Classification," IEEE Transactions on Circuits and Systems for Video Technology, vol. 17, no. 3, p. 372, 2007

[14] Q. Li, J. Ye, and C. Kambhamettu, "Linear Projection Methods in Face Recognition under Unconstrained Illuminations: A Comparative Study," in IEEE Computer Society Conference on Computer Vision and Pattern Recognition, vol. 2. IEEE Computer Society; 1999, 2004.

[15] S. Raudys and A. Jain, "Small sample size effects in statistical pattern recognition: recommendations for practitioners,"
Pattern Analysis and Machine Intelligence, IEEE Transactions on, vol. 13, no. 3, pp. 252-264, 1991.

[16] W. Zhao, R. Chellappa, and A. Krishnaswamy, "Discriminant analysis of principal components for face recognition," in Automatic Face and Gesture Recognition, 1998. Proceedings. Third IEEE International Conference on, 1998, pp. 336-341.

[17] P. Noel, "Golf swing training device," Feb. 22 1994, uS Patent $5,288,073$.

[18] http://www.homeswingtrainer.com/, "Home Swing Trainer."

[19] R. Jafari, W. Li, R. Bajcsy, S. Glaser, and S. Sastry, "Physical Activity Monitoring for Assisted Living at Home," in IFMBE Proceedings, vol. 13. Springer, 2007, p. 213.

[20] H. Ghasemzadeh, E. Guenterberg, K. Gilani, and R. Jafari, "Action coverage formulation for power optimization in body sensor networks," in Design Automation Conference, 2008. ASPDAC 2008. Asia and South Pacific, 2008, pp. 446-451.

[21] H. Ghasemzadeh, J. Barnes, E. Guenterberg, and R. Jafari, "A Phonological Expression for Physical Movement Monitoring in Body Sensor Networks," in Mobile Ad Hoc and Sensor Systems, 2008. MASS 2008. 5th IEEE International Conference on, 2008, pp. 58-68.

[22] H. Ghasemzadeh, E. Guenterberg, and R. Jafari, "Energy-Efficient Information-Driven Coverage for Physical Movement Monitoring in Body Sensor Networks," IEEE Journal on Selected Areas in Communications, vol. 27, pp. 58-69, 2009.

[23] M. Mathie, B. Celler, N. Lovell, and A. Coster, "Classification of basic daily movements using a triaxial accelerometer," Medical and Biological Engineering and Computing, vol. 42, no. 5, pp. 679-687, 2004.

[24] R. Ramachandran, L. Ramanna, G. Hassan, G. Pradhan, R. Jafari, and B. Prabhakaran, "Body Sensor Networks to Evaluate Standing Balance: Interpreting Muscular Activities Based on Inertial Sensors," in HealthNet'08: Proceedings of the 2st ACM SIGMOBILE international workshop on Systems and networking support for healthcare and assisted living environments. ACM, 2008.

[25] D. Spelmezan and J. Borchers, "Real-time snowboard training system," 2008.

[26] R. Gonzalez, D. Alvarez, A. Lopez, and J. Alvarez, "Modified Pendulum Model for Mean Step Length Estimation," in Engineering in Medicine and Biology Society, 2007. EMBS 2007. 29th Annual International Conference of the IEEE, 2007, pp. 1371-1374.

[27] J. Alvarez, R. Gonzalez, D. Alvarez, A. Lopez, and J. Rodriguez-Uria, "Multisensor Approach to Walking Distance Estimation with Foot Inertial Sensing," in Engineering in Medicine and Biology Society, 2007. EMBS 2007. 29th Annual International Conference of the IEEE, 2007, pp. 5719-5722

[28] R. Urtasun, D. Fleet, and P. Fua, "Monocular 3-D Tracking of the Golf Swing," in Computer Vision and Pattern Recognition, 2005. CVPR 2005. IEEE Computer Society Conference on, vol. 2, 2005.

[29] I. Karliga and J. Hwang, "Analyzing Human Body 3-D Motion of Golf Swing From Single-Camera Video Sequences," in Acoustics, Speech and Signal Processing, 2006. ICASSP 2006 Proceedings. 2006 IEEE International Conference on, vol. 5, 2006.

[30] A. Baca and P. Kornfeind, "Rapid Feedback Systems for Elite Sports Training," IEEE Pervasive Computing, pp. 70-76, 2006

[31] N. Betzler, S. Kratzenstein, F. Schweizer, K. Witte, and G. Shan, "3D Motion Analysis of Golf Swings." Poster at the 9 thInternational Symposium on the 3D Analysis of Human Movement. Valenciennes, France, 2006.

[32] K. Teu and F. Fuss, "Using dual number method for motion analysis of left arm in a golf swing," in Proceedings of the 2004 ACM SIGGRAPH international conference on Virtual Reality continuum and its applications in industry table of contents. ACM New York, NY, USA, 2004, pp. 217-220.

[33] K. King, S. Yoon, N. Perkins, and K. Najafi, "Wireless MEMS inertial sensor system for golf swing dynamics," Sensors $\&$ Actuators: A. Physical, vol. 141, no. 2, pp. 619-630, 2008.

[34] J. Doss, "Swing guide for golfer," Jul. 3 2007, uS Patent $7,238,117$.

[35] R. Kuster, "Golf swing training device," Nov. 20 2003, uS Patent App. 10/717,841.

[36] J. Lane, "Golf swing training method," Feb. 15 2000, uS Patent $6,024,656$. 